\title{
Real Time Health Monitoring System Using Arduino with Cloud Technology
}

\author{
R. Anandh ${ }^{1}$ and G. Indirani ${ }^{2}$ \\ ${ }^{1}$ Research Scholar, ${ }^{2}$ Assistant Professor, \\ ${ }^{1 \& 2}$ Department of Computer Science and Engineering, Annamalai University, Tamil Nadu, India \\ E-Mail: raanandh37@gmail.com, induk0992@gmail.com
}

\begin{abstract}
Internet of Things (IoT) is a new technology in which smart devices are connected to the internet. IoT serves and motivates for health monitoring systems where sensors that are either worn inside or outside the human body. The sensors collect the information of physical and logical conditions and the movements of the patient. This research paper aims to develop a system which gives body temperature and heart rate using sensors LM35 and $A D 8232$ heart rate monitor sensor respectively. These sensors are incorporated and interfaced with Arduino UNO board, ESP8266 for wireless data transmission to Thing Speak. The outcome of result on Thing speak is Data visualization. So that record of data can be stored and accessed over a period of time.
\end{abstract}

Keywords: IOT, Health Monitoring, Heart Rate, Body Temperature, ARDUINO, Thing Speak

\section{INTRODUCTION}

The modern healthcare provides a better caretaker for the patient worldwide with reasonable cost to improve patient monitoring devices. A new device designed to improve health monitoring using a microcontroller and biomedical sensors like temperature sensor, body rate sensors used for monitoring the health condition of the patient health condition on a single chip. If any changes takes place the data will be updated on the cloud, this notification helps to take an appropriate action at an instance of time, it can help for the patient's future health problem which arises. It also helps the patient's concern doctor to take an appropriate action at a proper time.

\section{OBJECTIVES OF THE STUDY}

The main objective of our paper is a remote health monitoring system using IOT and the data can be stored in the cloud. The data can be retrieved from the cloud over the internet. The system incorporates sensors that generate data and the information is collected from different sensors and the collected data sent to the cloud. The data can be analysed and maintained by the doctors. A database is to be maintained by the centralized server so the data can be retrieved from the patient's previous record history and provide better medical treatment. This digital output can be connected to microcontroller directly to measure the Beats per Minute (BPM) rate. It works on the principle of light modulation by blood flow through finger at each pulse. Various other sensors like the blood pressure sensor, Heartbeat sensor, temperature sensor, can be added to the patient kit in response to the patient's medical condition. Finally, IoT based Health Monitoring System which records the patient heartbeat rate and body temperature and also an email/SMS alert can be sent whenever those readings go beyond critical values. Patient pulse per minutes and temperature data can be monitored and recorded over Thing Speak. So the patient's medical history can be monitored from anywhere in the world over the internet.

\section{METHODOLOGY}

Our proposed design uses two sensors one is temperature sensor (LM35) another one is the pulse rate sensor (AD 8232). The Block diagram of Health Monitoring system is shown in Fig.1.

\section{A. Block Diagram}

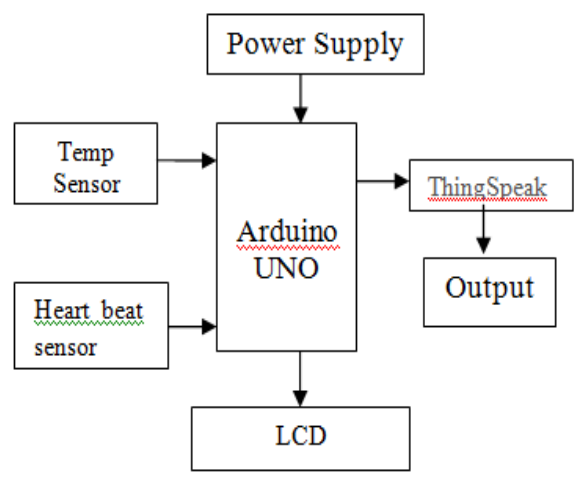

Fig. 1 Block diagram of health monitoring system

Here two sensors are used to collect the data and collected data put in the cloud through Thing Speak. The collected data are also displayed through Liquid Crystal Display (LCD).

\section{B. Hardware}

\section{Pulse Rate Sensor}

The pulse sensor is ready for use immediately connected and designed for Arduino. The top of the sensor is like heart shape, this side makes contact with our body like skin. On the front side, a round hole shape where the LED glows 
from the back. Under the LED an ambient light sensor is available it looks like a square shape. The LED glows light into fingertip or other skin tissues and the sensor monitors and reads the amount of light sensor return, it shows to calculate the heart Pulse rate.

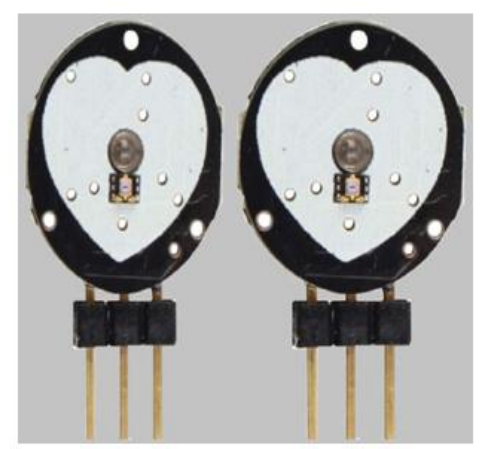

Fig. 2 Pulse rate sensor

Before using this sensor, it is required to protect the exposed side of the sensor so that accurate readings can be obtained and avoid the short circuit due to sweat. For this, a Velcro strip or black tape can be used.

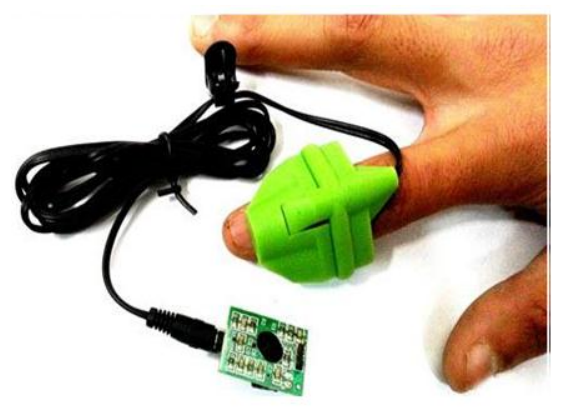

Fig. 3 Heart Beat Sensor AD8232

\section{LM35 Temperature Sensor}

LM35 is an analog linear temperature sensor. Its output is proportional to the temperature (in degree Celsius). The operating temperature range is from $-55^{\circ} \mathrm{C}$ to $150^{\circ} \mathrm{C}$. The output voltage varies by $10 \mathrm{mV}$ in response to every ${ }^{\circ} \mathrm{C}$ rise or fall in temperature. It can be operated from a $5 \mathrm{~V}$ as well as $3.3 \mathrm{~V}$ supply and the standby current is less than $60 \mathrm{uA}$.

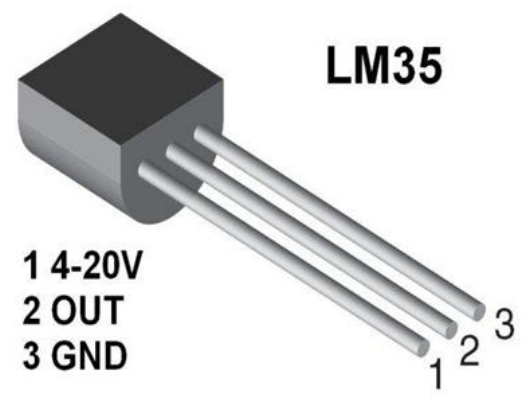

Fig. 4 Temperature sensor LM35

\section{ESP8266-01}

The ESP8266 microcontroller integrates a Tensilica L106 32-bit RISC processor, which achieves extra-low power consumption and reaches a maximum clock speed of 160 MHz. The Real-Time Operating System (RTOS) and Wi-Fi stack allow the processing power to be available for user application programming and development. It is integrated with a 32-bit Tensilica processor, standard digital peripheral interfaces, antenna switches, power amplifier, low noise receive amplifier, filters and power management modules. All of them are included in one small package; ESP8266 achieves low power consumption with a combination of several proprietary technologies. The power-saving architecture features three modes of operation: active mode, sleep mode and deep sleep mode. This allows batterypowered designs to run longer. ESP8266 is capable of functioning consistently in industrial environments, due to its wide operating temperature range. With highlyintegrated on-chip features and minimal external discrete component count, the chip offers reliability, compactness and robustness.

\section{Arduino Board}

The Arduino Uno is a microcontroller board based on the ATmega328. It has 14 digital input/output pins (of which 6 can be used as PWM outputs), 6 analog inputs, a $16 \mathrm{MHz}$ ceramic resonator, a USB connection, a power jack, an ICSP header, and a reset button. It contains everything needed to support the microcontroller; simply connect it to a computer with a USB cable or power it with a AC-to-DC adapter or battery to get started. The Uno differs from all preceding boards in that it does not use the FTDI USB-toserial driver chip. Instead, it features the Atmega16U2 programmed as a USB-to-serial converter. With the help of this it is possible to communicate with the PC or computer. "Uno" means one in Italian and is named to mark the upcoming release of Arduino 1.0. Arduino board has inbuilt $\mathrm{ADC}$ so there is no need to interface external ADC to connect with sensor, since most of the sensor gives their output in analog form. This board is also simple for programming it does not need any external programmer or burner to burn the program in microcontroller. Since it has $32 \mathrm{~KB}$ flash memory, the user's program can be saved and can also be modified according to the requirement.

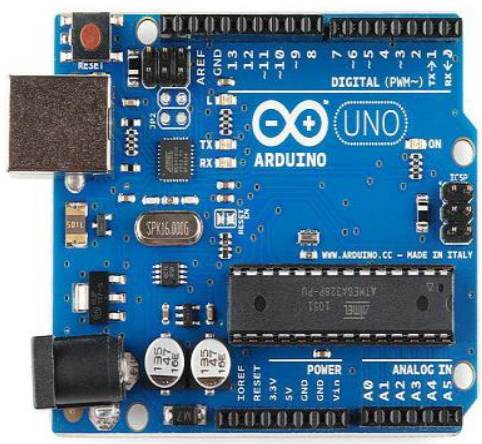

Fig. 5 Arduino Uno Board 


\section{Software}

Here the temperature and Heart beat rate are measured using the corresponding sensors. The sensed data are given as input to the Arduino uno microcontroller. If the values are above the threshold then alert message is given to the relatives of the patients. If the values measured from the sensors are below the threshold value, they are displayed in the LCD. Thing Speak is a free web service that lets to collect and store sensor data in the cloud and develop internet of things applications. The Thing Speak web service provides apps that let to analyse and visualize the data. Sensor data can be also be visualized using the serial plotter of the Arduino.

\section{UPLOADING THE DATA TO THE CLOUD}

After collecting the information from the sensors they need to be sent to cloud where it can be stored and displayed. Database of this application is also connected to the cloud.

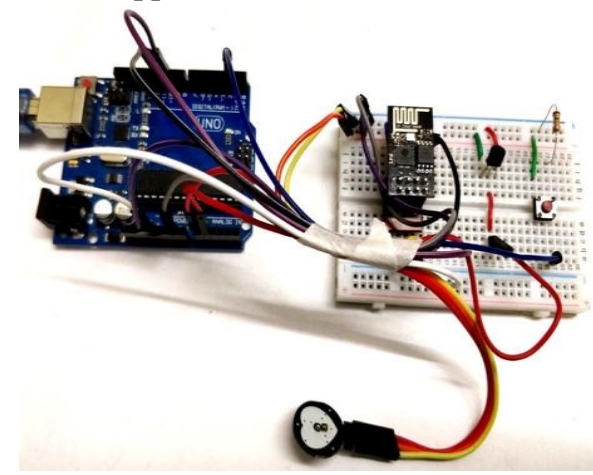

Fig. 6 IoT based Patient Monitoring System using ESP8266 and Arduino

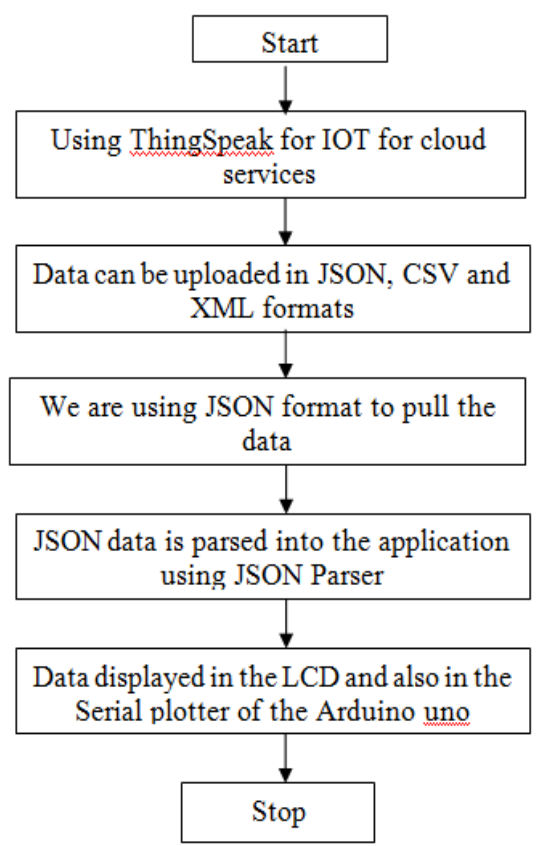

Fig. 7 Flow diagram for uploading the data in the cloud

\section{EXPERIMENTAL RESULTS}

Here particular threshold value is fixed for each sensor and if there is any change in the reading, it is displayed in the LCD screen and also updates in the cloud.

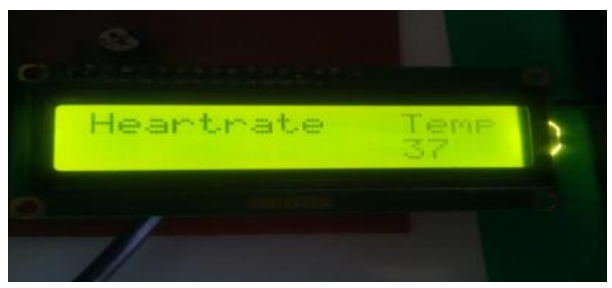

Fig. 8 Temperature display on LCD

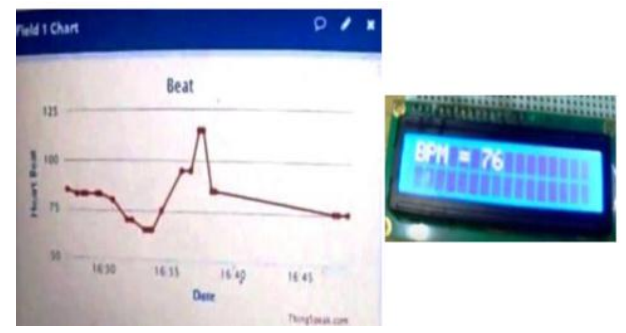

Fig. 9 Heart beat rate display on LCD and in the serial plotter

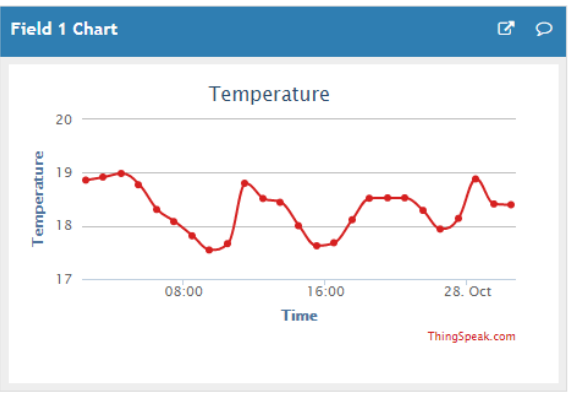

Fig. 10 Temperature result from the serial plotter

\section{CONCLUSION}

As a conclusion, a prototype of Health monitoring system using IoT has been successfully developed. In this model patient information are collected using various sensors and the collected data are put in the cloud through Thing Speak. The collected data are also displayed through LCD. Doctors can access the data from anywhere and there is no problem even if patients forgot to bring their report while consulting a physician.

\section{FUTURE WORK}

The future scope of this project is to integrate all the sensors into single chip by the use of fabrication in Nano scale and by making it as bio patch that can be attached to the skin and transmit the data through wireless medium. From the evaluation and the result obtained from analysis, the system is better for patients and also for the doctors to improve their patient's medical evaluation. 


\section{REFERENCES}

[1] I. Lee and K. Lee, "The internet of things (IoT): Applications, investments, and challenges for enterprises," Business Horizons, Vol. 58, No. 4, pp. 431-440, 2015.

[2] F. Cicirelli, G. Fortino, A. Giordano, A. Guerrieri, G. Spezzano, and A. Vinci, "On the design of smart homes: A framework for activity recognition in home environment," Journal of medical systems, Vol. 40, No. 9, pp. 1-17, 2016.

[3] P. Gope and T. Hwang, "Bsn-care: a secure iot-based modern healthcare system using body sensor network," IEEE Sensors Journal, Vol. 16, No. 5, pp. 1368-1376, 2016.

[4] E. Jovanov and A. Milenkovic, "Body area networks for ubiquitous healthcare applications: opportunities and challenges," Journal of medical systems, Vol. 35, No. 5, pp. 1245-1254, 2011.

[5] R. Gravina, P. Alinia, H. Ghasemzadeh, and G. Fortino, "Multisensor fusion in body sensor networks: State-of-the-art and research challenges," Information Fusion, Vol. 35, pp. 68-80, 2017.
[6] C. C. Poon, B. P. Lo, M. R. Yuce, A. Alomainy, and Y. Hao, "Body sensor networks: In the era of big data and beyond," IEEE reviews in biomedical engineering, Vol. 8, pp. 4-16, 2015.

[7] M. R. Yuce, "Implementation of wireless body area networks for healthcare systems," Sensors and Actuators A: Physical, Vol. 162, No. 1, pp. 116-129, 2010.

[8] G. Fortino, G. Di Fatta, M. Pathan, and A. V. Vasilakos, "Cloudassisted body area networks: state-of-the-art and future challenges," Wireless Networks, Vol. 20, No. 7, pp. 1925-1938, 2014.

[9] G. Aloi, G. Caliciuri, G. Fortino, R. Gravina, P. Pace, W. Russo, and C. Savaglio, "Enabling iot interoperability through opportunistic smartphone-based mobile gateways," Journal of Network and Computer Applications, Vol. 81, pp. 74-84, 2017.

[10] M. M. Hassan, K. Lin, X. Yue, and J. Wan, "A multimedia healthcare data sharing approach through cloud-based body area network," Future Generation Computer Systems, Vol. 66, pp. 48-58, 2017. 